\title{
Scheduling Algorithm for Improved System Capacity of Real-Time Services in 3GPP LTE
}

\author{
F. Rafael M. Lima, Walter C. Freitas and F. Rodrigo P. Cavalcanti
}

\begin{abstract}
Resumo-O foco deste artigo é em escalonamento de recursos para sistemas sem-fio multi-portadoras e com chaveamento por pacotes, tal como LTE (do inglês, Long Term Evolution). Nós propomos um escalonador chamado SORA-RT (do inglês, Satisfaction Oriented Resource Allocation - Real Time) que é projetado para garantir uma boa qualidade de serviço para aplicações de tempo real, tal como VoIP (do inglês, Voice over IP). SORA-RT é dividido em duas partes: Alocação de Recursos e Assinalamento de Recursos. Alocação de Recursos é responsável por selecionar os usuários para transmissão baseado na satisfação atual dos usuários, qualidade do canal sem-fio e parâmetros do serviço. Na parte de Assinalamento de Recursos é definido que recursos os usuários selecionados vão usar para transmissão de dados. Apresentamos também resultados obtidos via simulações computacionais da aplicação do escalonador SORA-RT no sistema LTE. Os resultados mostram que SORA-RT apresenta melhor desempenho que escalonadores clássicos encontrados na literatura quanto à garantia da qualidade de serviço para os usuários do serviço VoIP.
\end{abstract}

Palavras-Chave - Escalonamento, serviços de tempo real, LTE, VoIP.

Abstract-In this article we propose a scheduling algorithm to be used in multicarrier and packet-switched wireless systems, such as Long Term Evolution (LTE), named Satisfaction Oriented Resource Allocation - Real Time (SORA-RT). Our proposed scheduler can deal with Real Time (RT) traffic where it provides improved Quality of Service (QoS) to the connected flows and consequently increases the system capacity. The SORA-RT scheduler is split into two parts: Resource Allocation and Resource Assignment. In the first part, we define the scheduled flows based on their current satisfaction status, channel quality state and QoS parameters. In the Resource Assignment stage, the selected flows get assigned system resources in an opportunistic way. In a case study for the Long Term Evolution (LTE) system we show that the SORA-RT provides better user satisfaction than reference schedulers in the literature for Voice over IP (VoIP) traffic.

Keywords-Scheduling, RT services, LTE, VoIP.

\section{INTRODUCTION}

Nowadays, many people can access data services using mobile phones due to the successfull deployment of Third Generation $(3 \mathrm{G})$ networks worldwide. However, the demand for new multimedia services, lower costs and improved Quality of Service (QoS) provision increases as quick as the evolution of mobile communications. Therefore, 3rd. Generation Partnership Project (3GPP) with other standardization bodies have been working in the specification of a new system called Long Term Evolution (LTE).

LTE promises to bring advantages for subscribers, with new applications such as interactive TV and user-generated videos,

Email addresses: \{rafaelm,walter,rodrigo\}@gtel.ufc.br and for operators with backward compatibility with legacy networks and simpler architecture. Among the main features of LTE we can state [1]:

- Downlink peak data rates over $200 \mathrm{Mbits} / \mathrm{s}$;

- Radio Access Network (RAN) round-trip times less than $10 \mathrm{~ms}$;

- Bandwidth flexibility ranging from less than $5 \mathrm{MHz}$ to $20 \mathrm{MHz}$;

- Support of both Frequency Division Duplex (FDD) and Time Division Duplexing (TDD) duplexing modes;

- Reduced number of physical and logical nodes.

Besides the presented LTE features, we highlight the utilization of Orthogonal Frequency Division Multiple Access (OFDMA) as the radio access technology in the downlink and a pure packet-based All-Internet Protocol (IP) architecture.

OFDMA is a multicarrier radio access technology that can improve the spectral efficiency compared to other schemes [2]. OFDMA is based on Orthogonal Frequency Division Multiplexing (OFDM) where multiple service flows ${ }^{1}$ get assigned subcarriers or subsets of them in order to be served simultaneously. One of the advantages of the multiple access scheme OFDMA is the opportunity to benefit from frequency and multiuser diversities [3]. Frequency diversity relies on the fact that the fading is different at different frequencies. Multiuser diversity is a form of diversity inherent in cellular wireless systems, provided by independent time-varying channels across the different terminals.

A mechanism for taking advantage of the frequency and multiuser diversities is the employment of scheduling algorithms. Scheduling algorithms are responsible for selecting which terminals will have access to the system radio resources and with which configuration. For OFDMA, due to the flexibility and higher resource granularity, scheduling plays an even more important role than in $3 \mathrm{G}$ systems.

An All-IP architecture allows the efficient support to mass-market usage of any IP-based service and reduced Operational Expenditure (OPEX), i.e., on going costs to run a network, and Capital Expenditure (CAPEX), i.e., costs to upgrade and expand the network. However, this architecture imposes some challenges on the provision of QoS guarantees of Real Time (RT) services, e.g., speech, that have been traditionally provided over circuit-switched connections. On circuit-switched connections, the system resources are allocated on the beginning of the connection

\footnotetext{
${ }^{1}$ A User Equipment (UE) can bear multiple service flows. Without loss of generality, in this study, only one service flow is considered per UE. Therefore, flow and UE are interchangeable throughout the text.
} 
and remain unchanged until the end of the connection. In an All-IP network, the RT flows have resources assigned in a packet-switched way, i.e., the resources are allocated dynamically according to the individual demands of the connections. Once again, the design of scheduling algorithms is of utmost concern. The scheduler should be able to guarantee that the packet delays are as short as possible.

Relevant works in the literature about scheduling algorithms and Voice over IP (VoIP) service are [4], [5], [6]. In [4] the authors evaluate the performance of the VoIP service in the High Speed Downlink Packet Access (HSDPA) system when other services are present. The work [5] was one of the first studies about VoIP service in the LTE system, and basically evaluates how general system parameters, such as antenna diversity, link adaptation and control channels, affect the performance of VoIP service. In [6] the authors proposed a scheduling algorithm to control the spectral innefficiency incurred when express priority is assigned to VoIP service.

In [7] we proposed the Satisfaction Oriented Resource Allocation - Non Real Time (SORA-NRT) scheduling algorithm that improves the user satisfaction for Non-Real Time (NRT) services (non-delay-sensitive services). The improvement of the user satisfaction is advantageous since it can be directly mapped on an increased operator's revenue. None of the mentioned articles about scheduling and LTE have explicitly designed scheduling algorithms with this objective. Therefore, in this present work, we complement [7] by proposing the Satisfaction Oriented Resource Allocation - Real Time (SORA-RT) that aims at maximizing the user satisfaction of RT services. The performance of SORA-RT is evaluated in a case study in the LTE system. The remaining of this article is organized as follows: section II describes the main features of LTE system; section III formally states the problem to be solved; the proposed scheduler SORA-RT is presented in section IV; and finally the simulation results and conclusions are shown in sections $\mathrm{V}$ and VI, respectively.

\section{LONG TERM Evolution System}

Basically, LTE is composed of the following physical network elements:

- User Equipment (UE): Mobile user terminals capable of accessing the wireless services;

- Enhanced Node B (eNB) or base station: Network element that serves the user terminals;

- Mobility Management Entity (MME) / Serving Gateway (S-GW): Network elements responsible for mobility management and user plane functions.

These network elements and their interfaces are illustrated in Fig. 1. MME is the key control node for Evolved UMTS Terrestrial Radio Access Network (E-UTRAN) and mainly perform the following functions: Non-Access Stratum (NAS) signaling, UE tracking in idle mode including control and execution of paging retransmissions, bearer management and S-GW selection. eNBs are responsible for selecting and routing data to/from the MME/S-GW and some Radio Resource Management (RRM) functionalities such as call admission control, Adaptive Modulation and Coding (AMC)

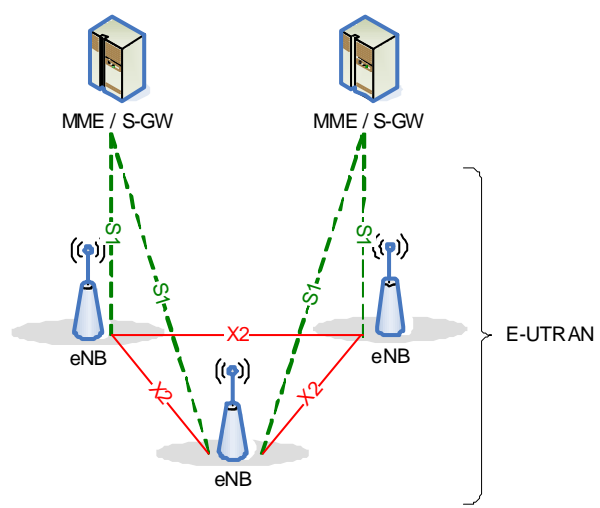

Fig. 1. LTE network elements and their interfaces [8].
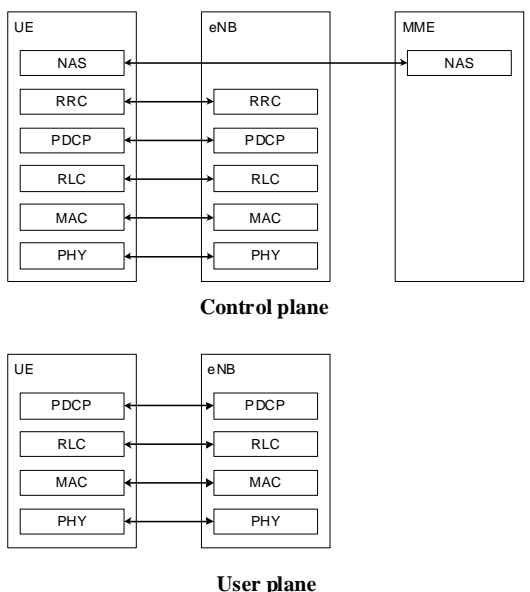

Fig. 2. User and control planes protocol stack [8]

and scheduling. Besides user data packet routing and forwarding, S-GW is the anchoring point for inter-eNB handover and inter-3GPP mobility. The eNBs are connected to MME/S-GW through the S1 interface and among each other through the $\mathrm{X} 2$ interface.

In Fig. 2, we show the protocol stack for user and control planes. In the control plane, the Radio Resource Control (RRC) protocol handles radio bearer setup, active mode mobility management and broadcast of system information. The NAS protocols deal with idle mode mobility management, service setup, authentication and security. The Packet Data Convergence Protocol (PDCP) layer performs ciphering and integrity check in the control plane. Regarding user plane, the PDCP layer is responsible for header compression of IP packets and ciphering. The Radio Link Control (RLC), Medium Access Control (MAC) and Physical (PHY) layers have the same function in the user and control planes. RLC layer focuses on lossless transmission of data, and MAC layer handles uplink and downlink scheduling, and Hybrid Automatic Repeat Request (HARQ) signaling. The PHY layer is responsible for protecting data against channel errors using AMC schemes based on channel conditions.

In the following we describe in more details the MAC and PHY layers that play an important role in scheduling. 


\section{A. Physical Layer}

LTE supports both FDD and TDD duplexing schemes. In this study FDD is utilized. The time domain structure of LTE is composed of radio frames of $10 \mathrm{~ms}$. Each radio frame has 10 equally-sized subframes of length $1 \mathrm{~ms}$. Subframes, in turn, consist of two slots of length $0.5 \mathrm{~ms}$. The scheduling takes place in a subframe basis [8].

The default subcarrier spacing is $15 \mathrm{kHz}$ and all subcarriers are grouped in sets of 12 subcarriers. A resource block in LTE is defined as a two-dimensional grid with 12 subcarriers in frequency and $0.5 \mathrm{~ms}$ in time that corresponds to 6 or 7 OFDM symbols depending on cyclic prefix length. A Resource Unit (RU) in the system is composed of two resource blocks concatenated in the time domain, i.e., 12 subcarriers and $1 \mathrm{~ms}$. The resource structure in LTE system is illustrated in Fig. 3. The physical resources are utilized by physical channels and signals. Physical channels are utilized for transmission of data and/or control information from the MAC layer. The physical signals are used to support physical-layer functionality and do not carry any information from the MAC layer [9].

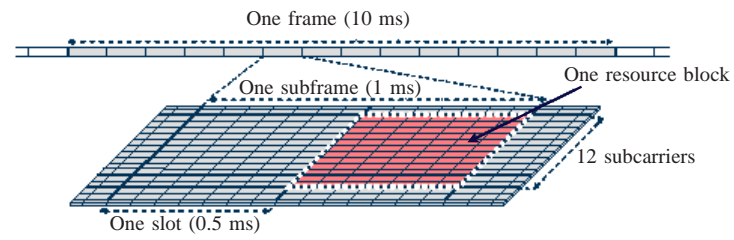

Fig. 3. Time frame and resource structure in LTE.

Among the physical channels, we highlight Physical Downlink Control Channel (PDCCH). This channel is used to carry uplink scheduling grants and downlink scheduling assignments, such as resource indication, transport format, HARQ information and transport block size. Depending on the time-variant PDCCH capacity, different number of UEs can be scheduled in a given Transmission Time Interval (TTI). However, the maximum number of scheduled UEs in a TTI was simplified to a fixed number over a cell in this study.

\section{B. Medium Access Control}

The MAC layer in LTE is responsible for HARQ, transport format selection, and priority handling (scheduling) [10].

The HARQ is modeled as a number of processes where each process uses a simple stop-and-wait protocol. HARQ for downlink is asynchronous and adaptive. By asynchronous we mean that the scheduler has the freedom to choose the subframe for retransmission dynamically. In adaptive HARQ, the scheduler can use a different resource for retransmission compared to the previous (re)transmission. The version redundancy of a (re)transmission needs to be known by the receiver in order to perform soft combination.

Due to the HARQ retransmissions, MAC Protocol Data Units (PDUs) can arrive at the receiver in a different order of the transmission, therefore, the MAC layer does not provide in-order delivery to the RLC layer. Finally, MAC performs multiplexing of RLC PDUs of different flows of a single UE.

\section{PROBLEM FORMULATION}

Consider a multicarrier system where the flows are from a RT service. The problem to maximize the user satisfaction ratio is

$$
\begin{aligned}
\max _{\mathbf{X}[k]} & \sum_{j \in \Omega} U(j, k) \\
& \text { subject to } \\
& \sum_{j \in \Omega} x_{j, n}[k] \leqslant 1, \quad \forall n \in \mathcal{N}, \\
& \sum_{j \in \Omega} \Psi\left(\sum_{n \in \mathcal{N}} x_{j, n}[k]\right) \leqslant S,
\end{aligned}
$$

where $\mathcal{N}$ is the RU set and $\Omega$ is the active flow set. We consider that active flows are the ones with packets awaiting transmission. $S$ is the maximum allowable number of scheduled UEs per TTI. $\mathbf{X}[k]$ is the assignment matrix with elements $x_{j, n}[k]$ defined as

$x_{j, n}[k]= \begin{cases}1, & \text { if RU } n \text { is assigned to flow } j \text { in the TTI } k \\ 0, & \text { otherwise. }\end{cases}$

$\Psi(g)$ is a step function that assumes 1 when $g$ is positive and 0 otherwise. $U(j, k)$ is the satisfaction function of flow $j$ at TTI $k$ and is defined as

$$
U(j, k)= \begin{cases}1, & \text { if } F E R_{j}[k] \leq F E R_{j}^{\text {req }} \\ 0, & \text { otherwise }\end{cases}
$$

where $F E R_{j}[k]$ is the accumulated Frame Erasure Rate (FER) for flow $j$ at TTI $k$, and $F E R_{j}^{\text {req }}$ is the FER requirement of flow $j . F E R_{j}[k]$ is defined as

$$
F E R_{j}[k]=\frac{n_{j}^{\text {lost }}[k]}{n_{j}^{\text {lost }}[k]+n_{j}^{\text {succ }}[k]} \forall j \in \Omega,
$$

where $n_{j}^{\text {succ }}[k]$ is the number of successfully transmitted packets from flow $j$ until TTI $k$ and $n_{j}^{\text {lost }}[k]$ is the number of lost packets from flow $j$ until TTI $k$.

The first constraint in problem (1) represents the system restriction that one RU cannot be allocated to more than one $\mathrm{UE}$ in a cell. In this way, no intra-cell interference is present. In the second constraint we model the limitation in the PDCCH capacity that imposes a restriction in the number of scheduled UEs per TTI.

Note that problem (1) is a non-linear combinatorial problem whose optimal solution is not easily found. Computational heavy algorithms to search for optimum solutions may not be suitable to the small time-scale with which the scheduling takes place in LTE system. For that reason, simple algorithms that provide sub-optimum and low-complexity solutions are highly recommended. In the next section we present the main contribution of this paper: the SORA-RT scheduler. 


\section{SORA-RT}

Both the SORA-NRT and SORA-RT schedulers have a common core that utilizes smart and simple heuristics. We have adopted the same strategy of splitting the RU allocation into two parts, as described in [7]. Thus, SORA-RT is composed of two building blocks: Resource Allocation and Resource Assignment, as shown in Fig. 4. The Resource Allocation part is responsible for defining which flows will be scheduled and determining an estimate of how many resources they will receive, while the Resource Assignment part defines which resources will be associated with which flow.

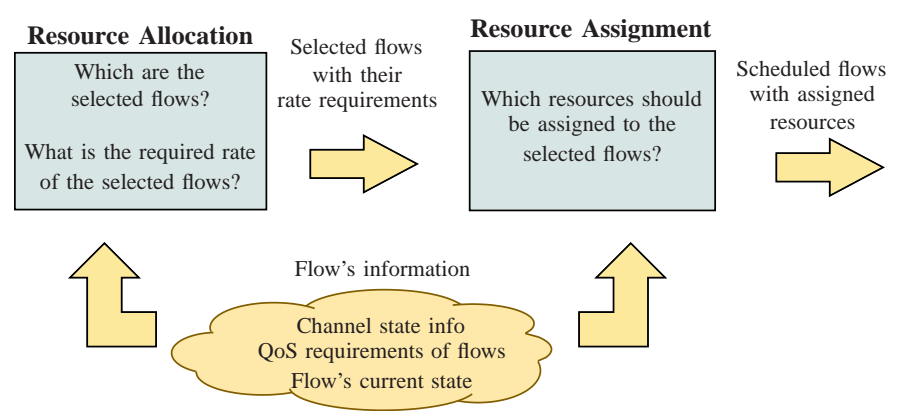

Fig. 4. Building blocks of SORA-RT.

\section{A. Resource Allocation}

The first step in the Resource Allocation part is to assign a priority for each flow. Before defining this priority we first introduce variable $d_{j}[k]$ as in the following

$$
\begin{cases}\left\lfloor\frac{n_{j}^{\text {total }}[k] \cdot F E R_{j}^{\text {req }}-n_{j}^{\text {lost }}[k]}{1-F E R_{j}^{\text {req }}}\right\rfloor, & \text { if } F E R_{j}[k] \leq F E R_{j}^{\text {req }} \\ \left\lceil\frac{n_{j}^{\text {lost }}[k]-n_{j}^{\text {total }}[k] \cdot F E R_{j}^{\text {req }}}{F E R_{j}^{\text {req }}}\right\rceil, & \text { otherwise, }\end{cases}
$$

where $\lfloor v\rfloor$ represents the first integer equal to or lower than $v$, $\lceil v\rceil$ is the first integer equal to or greater than $v$, and $n_{j}^{\text {total }}[k]=$ $n_{j}^{\text {succ }}[k]+n_{j}^{\text {lost }}[k]$ is the total number of generated packets of flow $j$ until TTI $k$.

$d_{j}[k]$ has a different meaning according to the satisfaction state of a flow. If a flow $j$ is currently unsatisfied $\left(F E R_{j}[k]>F E R_{j}^{\text {req }}\right) \quad d_{j}[k]$ represents the number of packets the flow $j$ should successfully transmit in a row so as to become satisfied. On the other hand, if the flow $j$ is currently satisfied $d_{j}[k]$ is the maximum number of packets that this flow can lose successively and still be satisfied. In other words, $d_{j}[k]$ can be seen as a distance that an unsatisfied (or satisfied) flow is from satisfaction (or dissatisfaction).

The prioritization is split into two levels: an outer and inner prioritization. In the outer prioritization the flows that have pending HARQ retransmissions have the highest priority. Moreover, the flows that are currently satisfied have precedence over the ones that are unsatisfied. The idea behind this last strategy is to guarantee that the satisfied flows will have resource enough to maintain the desired QoS. Within the group of satisfied, unsatisfied and with pending retransmission we define the inner priority $p_{j}[k]$ as

$$
\begin{cases}D_{j}^{\text {oldest }}[k], & \text { if flow } j \text { is in } \mathrm{rtx} \\ \frac{1}{\left(D_{j}^{r e q}-D_{j}^{\text {oldest }}[k]\right) \cdot\left(d_{j}[k]+1\right)}, & \text { otherwise, }\end{cases}
$$

where $D_{j}^{r e q}$ and $D_{j}^{\text {oldest }}[k]$ are the packet delay requirement and current packet delay of the oldest packet of flow $j$, respectively. We can see that the inner priority gives precedence to the flows that are either close to the satisfaction or dissatisfaction states and whose oldest packet is close to its deadline. Fig. 5 summarizes the outer and inner prioritization.

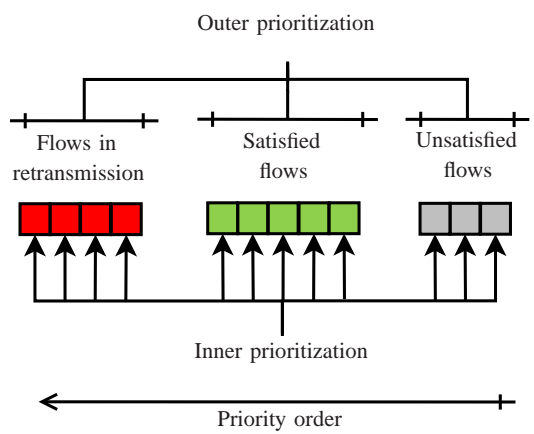

Fig. 5. Illustration of the prioritization within SORA-RT.

Once the priority among flows is defined the Resource Allocation part selects the first more prioritized $S$ flows to have transmission opportunity in the Resource Assignment. The final step in the Resource Allocation part is to define the required rate for the selected flows, $\Delta r_{j}[k]$. This metric is used in the Resource Assignment part to control the amount of RUs that will be assigned to the selected flows. It is defined as

$$
\Delta r_{j}[k]=\frac{b_{j}^{\text {oldest }}[k]}{T_{0}} \text { for all selected } j,
$$

where $b_{j}^{\text {oldest }}[k]$ represents the number of bits of the oldest packet from flow $j$ at TTI $k$ and $T_{0}$ is the time length of a TTI.

\section{B. Resource Assignment}

The Resource Assignment part is performed in assignment phases so that in each phase a flow can get only one resource. In the beginning of a new phase the flows are prioritized according to their best RU, i.e., the flow with the RU in better channel quality among all RUs of all flows gets its RU first. The flows compete for resources in this part until their data rate requirements defined in the Resource Allocation part are fulfilled. In case all flows achieve the required rate and there are still unused resources, the resource assignment starts again in the same way as described.

\section{RESUlTS}

This section is devoted to the performance evaluation of SORA-RT in a case study with LTE. The first three subsections present the main aspects regarding the simulation environment and modeling. The results are presented in the subsection V-D 


\section{A. Simulation Setup}

The results presented in this section are drawn from a dynamic system-level simulator that models the LTE system according to 3GPP specifications [8]. In this simulator, the main assumptions and restrictions of a real LTE system are modeled.

The simulator model includes multiple cells, inter-cell interference and propagation phenomena such as path loss, shadowing and fast fading. Other important aspects related to LTE were modeled, such as the radio interface protocols MAC, RLC and PDCP, and higher protocol layers, such as Transport Control Protocol (TCP), User Datagram Protocol (UDP), IP and applications. The main simulation parameters are shown in TABLE I.

TABLE I

SIMULATION PARAMETERS.

\begin{tabular}{|c|c|}
\hline Parameter & Value \\
\hline \multicolumn{2}{|l|}{ LTE network } \\
\hline Bandwidth/Number of RUs & $3 \mathrm{MHz} / 15$ \\
\hline Carrier frequency & $2 \mathrm{GHz}$ \\
\hline Total cell power & $20 \mathrm{~W}$ \\
\hline $\begin{array}{c}\text { Transport network packet delay } \\
\text { (including Internet and Core Network }(\mathrm{CN}) \text { ) }\end{array}$ & $14 \mathrm{~ms}$ \\
\hline Maximum number of scheduled terminals per TTI & 5 \\
\hline \multicolumn{2}{|l|}{$\begin{array}{ll} & \text { Propagation } \\
\end{array}$} \\
\hline Path gain at 1 meter distance & $-29.03 \mathrm{~dB}$ \\
\hline Path gain per dB distance & $-3.52 \mathrm{~dB}$ \\
\hline Shadowing standard deviation & $8 \mathrm{~dB}$ \\
\hline Antenna type & SCM 3GPP [11] \\
\hline \multicolumn{2}{|l|}{ Deployment } \\
\hline Number of eNBs/cells per eNB & $3 / 3$ \\
\hline Number of antennas in the terminal/cell & $2 / 1$ \\
\hline Cell radius & $500 \mathrm{~m}$ \\
\hline Frequency reuse & $1 / 3$ \\
\hline Fast fading speed & $3 \mathrm{~km} / \mathrm{h}$ \\
\hline \multicolumn{2}{|l|}{ VoIP Service } \\
\hline Number of HARQ processes & 16 \\
\hline Maximum number of HARQ retransmissions & 10 \\
\hline RLC Service Data Unit (SDU) discard period & $80 \mathrm{~ms}$ \\
\hline Mean talk period time & $5 \mathrm{~s}$ \\
\hline Voice activity factor & 0.5 \\
\hline Frame size & 264 bits \\
\hline Frame period & $20 \mathrm{~ms}$ \\
\hline Maximum end-to-end VoIP frame delay & $140 \mathrm{~ms}$ \\
\hline Silence Insertion Description (SID) frame size & 39 bits \\
\hline Required FER & $1 \%$ \\
\hline VoIP satisfaction threshold & $95 \%$ \\
\hline
\end{tabular}

\section{B. Definition of Performance Metrics}

The VoIP packet delay is the time length since the transmission of the VoIP frame from the user behind the internet and the reception of the frame at the terminal. The VoIP FER is defined in equation 4. A packet is lost if it does not arrive at the receiver because of an RLC SDU discard at the transmitter or an HARQ failure, or its reception is performed with a delay greater than the maximum VoIP packet delay. A VoIP flow is considered satisfied if its FER is lower

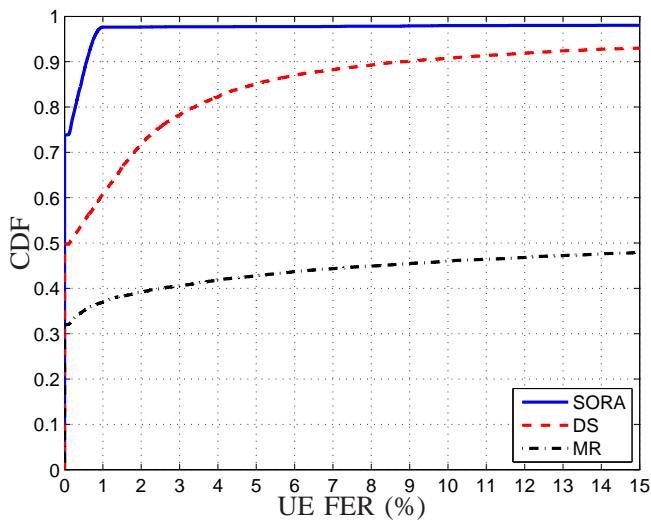

Fig. 6. VoIP FER CDF at load 289 UEs per cell.

than or equal to the required FER. Finally, the play-out buffer at the receiver is configured in such a way that it virtually eliminates the delay jitter of VoIP packets received within the delay budget.

Regarding cell/system measurements, the user satisfaction ratio is defined as the ratio between the number of satisfied flows and the total number of flows [12].

\section{Reference Schedulers}

We compare the performance of the SORA-RT scheduler with two reference schedulers: Delay Scheduler (DS) and Maximum Rate (MR). DS prioritizes the flow whose headline RLC SDU has the current greatest delay. The MR scheduler chooses the flow that can transmit more information bits when using the available bandwidth (better channel condition).

In all these schedulers, the selected flow receives the necessary RUs to transmit all pending RLC SDUs. The RUs are selected in an opportunistic way, i.e., the terminal gets assigned its best RUs. Another flow is scheduled only if the first selected flow does not need all the RUs to transmit its data.

\section{Performance Results}

We start showing in Fig. 6 the Cumulative Distribution Function (CDF) of the VoIP FER at a specific load of 289 flows per cell in order to illustrate how SORA-RT performs a QoS control. Firstly, we can see that the MR scheduler provides higher values for FER which leads to a poor user satisfaction. The first reason for this is the fact that MR does not take into account the current packet delay in its formulation. The other reason is the selection criterion of MR. Since it selects the flows in better channel conditions, these flows need few RUs to transmit the buffered data. However, due to the limitation in the number of scheduled flows in a TTI, many RUs remain unused after the scheduling process.

DS achieves better FERs than MR scheduler. In fact, DS prioritizes flows with high packet delays. Therefore, packets with low delays have to wait in the transmit buffer until becoming an urgent packet and then be transmitted. This is a smart strategy when VoIP service is regarded since VoIP 


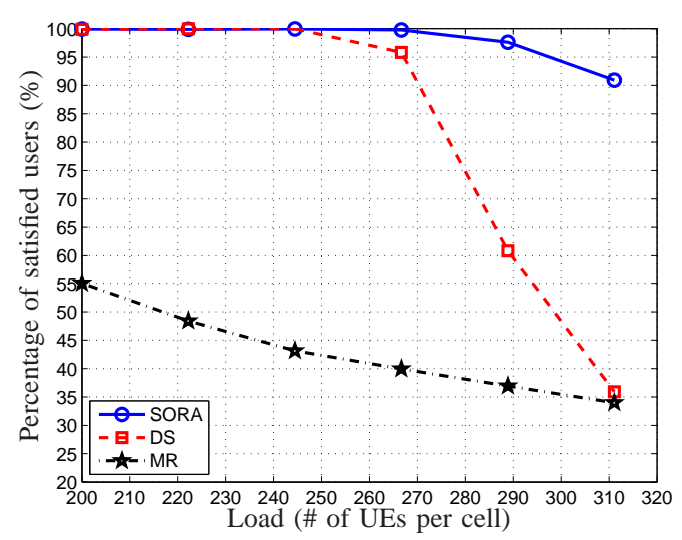

Fig. 7. User satisfaction ratio versus system load.

packets that arrive at the receiver with delays lower than a maximum tolerable delay are still useful. In this way, by transmitting packets later but within the maximum delay threshold, more successful packets can arrive at the receiver and packet discard at the transmitter occurs less often leading to low FER.

Another reason to prioritize flows with high headline packet delays is that these flows usually have more than one buffered packet to transmit. The transport block size in LTE utilizing one RU can, depending on the modulation order and code rate, be greater than one RLC SDU that is mapped one to one with VoIP frames. As a result, scheduling flows with high headline packet delays increases the efficiency by reducing the protocol layer overheads and padding rate per sent VoIP packet [5].

Despite the better performance of DS compared to MR scheduler, the FER is even improved with SORA-RT. As it can be seen, most of the FERs provided by SORA-RT scheduler are concentrated below the FER threshold of $1 \%$. This is one of the key factors to provide a high number of satisfied users. It occurs because SORA-RT besides prioritizing VoIP flows with larger delays, it also preempts the flows that have an excessively good QoS to give transmission opportunities to the flows that have critical packets to transmit.

In Fig. 7 we show the user satisfaction ratio. The poor performance of MR scheduler is confirmed in this figure. The advantage of taking into account packet delay when scheduling VoIP traffic can be represented by the performance gain obtained of DS over MR. However, SORA-RT overperforms DS which shows that the user satisfaction can be even improved. With a satisfaction threshold of $95 \%$, the number of flows supported in a cell with SORA-RT scheduler is about 297, which is 30 flows more than the number of supported flows when DS is used. This gain comes from the smart strategy of SORA-RT to perform a QoS balance in order to guarantee that a high number of flows achieve the minimum acceptable QoS.

\section{CONCLUSIONS}

This work presents the Satisfaction Oriented Resource Allocation - Real Time (SORA-RT) scheduler that is a downlink scheduler to be utilized in multicarrier networks to improve the user satisfaction of Real Time (RT) services. The SORA-RT scheduler is designed to benefit from the main characteristics of multicarrier schemes, such as the multiuser and frequency diversities. SORA-RT is split into two parts: Resource Allocation and Resource Assignment. In the first part, the flows that will be scheduled are defined based on their current satisfaction status, channel quality state and Quality of Service (QoS) parameters. In the second part, the selected flows get assigned system resources in an opportunistic way.

In a case study with Voice over IP (VoIP) service in the 3rd. Generation Partnership Project (3GPP)'s Long Term Evolution (LTE) system, the SORA-RT scheduler is able to provide a capacity gain of $11 \%$ over a well known scheduler for RT traffic.

\section{ACKNOWLEDGEMENT}

This work was supported by the Research and Development Centre, Ericsson Telecomunicações S.A., Brazil, under EDB/UFC.18 Technical Cooperation Contract. We thank FUNCAP for the financial support of F. R. M. Lima.

\section{REFERENCES}

[1] 3GPP, "Requirements for Evolved UTRA (E-UTRA) and Evolved UTRAN (E-UTRAN)," $3^{\text {rd }}$ Generation Partnership Project, Tech. Rep. TR 25.913 V8.0.0 - Release 8, Dezembro 2008.

[2] M. Sternad, T. Svensson, T. Ottosson, A. Ahlen, A. Svensson, and A. Brunstrom, "Towards Systems Beyond 3G Based on Adaptive OFDMA Transmission," Proceedings of the IEEE, vol. 95, no. 12, pp. 2432-2455, Dezembro 2007.

[3] R. Knopp and P. A. Humblet, "Information Capacity and Power Control in Single-Cell Multiuser Communications," in Communications, 1995. ICC '95 Seattle, 'Gateway to Globalization', 1995 IEEE International Conference on, vol. 1, Junho 1995, pp. 331-335.

[4] M. Ericson and S. Wanstedt, "Mixed Traffic HSDPA Scheduling Impact on VoIP Capacity," in Vehicular Technology Conference, 2007. VTC2007-Spring. IEEE 65th, Dublin, Abril 2007, pp. 1282-1286.

[5] F. Persson, "Voice over IP Realized for the 3GPP Long Term Evolution," in Vehicular Technology Conference, 2007. VTC-2007 Fall. 2007 IEEE 66th, Setembro 2007, pp. 1436-1440.

[6] S. Choi, K. Jun, Y. Shin, S. Kang, and B. Choi, "MAC Scheduling Scheme for VoIP Traffic Service in 3G LTE," in Vehicular Technology Conference, 2007. VTC-2007 Fall. 2007 IEEE 66th, Outubro 2007, pp. 1441-1445.

[7] F. R. M. Lima, R. B. dos Santos, F. Cavalcanti, and W. C. Freitas, "Radio Resource Allocation for Maximization of User Satisfaction," in Signal Processing Advances in Wireless Communications, 2008. SPAWC 2008. IEEE 9th Workshop on, Recife, Julho 2008, pp. 565-569.

[8] 3GPP, "Evolved Universal Terrestrial Radio Access (E-UTRA) and Evolved Universal Terrestrial Radio Access Network (E-UTRAN); Overall Description," $3^{\text {rd }}$ Generation Partnership Project, Tech. Rep. TS 36.300 V8.7.0 - Release 8, Dezembro 2008.

[9] — , "Evolved Universal Terrestrial Radio Access (E-UTRA); Long Term Evolution (LTE) Physical Layer; General Description," $3^{\text {rd }}$ Generation Partnership Project, Tech. Rep. TS 36.201 V8.2.0 - Release 8, Dezembro 2008.

[10] _ "Evolved Universal Terrestrial Radio Access (E-UTRA); Medium Access Control (MAC) Protocol Specification," $3^{\text {rd }}$ Generation Partnership Project, Tech. Rep. TS 36.321 V8.4.0 - Release 8, Dezembro 2008.

[11] _ , "Spatial Channel Model for Multiple Input Multiple Output (MIMO) Simulations," 3rd. Generation Partnership Project, Tech. Rep. TR 25.996 V8.0.0, Dezembro 2008.

[12] A. Furuskär, "Radio Resource Sharing and Bearer Service Allocation for Multi-Bearer Service, Multi-Access Wireless Networks," Ph.D. dissertation, Royal Institute of Technology (KTH), Radio Communication Systems, Abril 2003. 